\title{
Hemodynamic features of offending vessels at neurovascular contact in patients with trigeminal neuralgia and hemifacial spasm
}

\author{
Toru Satoh, MD, ${ }^{1}$ Takanobu Yagi, PhD, ${ }^{2}$ Keisuke Onoda, MD, ${ }^{3}$ Masahiro Kameda, MD, ${ }^{4}$ \\ Tatsuya Sasaki, MD, ${ }^{4}$ Tomotsugu Ichikawa, MD, ${ }^{4}$ and Isao Date, MD ${ }^{4}$ \\ ${ }^{1}$ Department of Neurological Surgery, Ryofukai Satoh Neurosurgical Hospital, Fukuyama, Hiroshima; ${ }^{2}$ Center for Advanced \\ Biomedical Sciences, Waseda University, Tokyo; ${ }^{3}$ Department of Neurological Surgery, Okayama Red Cross General Hospital; \\ and ${ }^{4}$ Department of Neurological Surgery, Okayama University Postgraduate Medical School, Okayama, Japan
}

\begin{abstract}
OBJECTIVE Offending vessels at the site of neurovascular contact (NVC) in patients with trigeminal neuralgia (TN) and hemifacial spasm (HFS) may have specific hemodynamic features. The purpose of this study was to investigate the wall shear stress (WSS) of offending vessels at NVCs by conducting a computational fluid dynamics (CFD) analysis.

METHODS The authors retrospectively analyzed the cases of 20 patients ( 10 with TN and 10 with HFS) evaluated by 3D $\mathrm{CT}$ angiography and used the imaging findings for analysis of the hemodynamic parameters. The 3D CFD images were directly compared with the NVCs determined by simulated multifusion images of CT angiogram and MR cisternogram, and operative photos. The magnitudes of the WSS (WSSm) at the proximal (WSSm-p), just-beginning (WSSm-j), contact site (WSSm-s), and distal (WSSm-d) areas of each NVC were analyzed. The ratios of the WSSm-j, WSSm-s, and WSSm-d areas to the WSSm-p area were calculated individually. The direction of the WSS (WSSv) and its temporal variation (WSSvV) were depicted and morphologically compared with the NVC confirmed by simulated images and operative findings.
\end{abstract}

RESULTS The ratios of WSSm at the just-beginning and the contact site to the proximal area of the NVCs (WSSm-j/ WSSm-p and WSSm-s/WSSm-p) were both significantly higher than that at the distal area (WSSm-d/WSSm-p) ( $p<$ 0.05). The WSSv and WSSVV at the NVCs showed small variation in a single cardiac cycle, especially along the areas that were in contact with the affected nerve.

CONCLUSIONS Areas of relatively high WSSm and temporal variation of WSSm (WSSmV) were observed at the NVCs. Less mobility of the WSSv and WSSvV was detected along the side of the vessels in contact with the nerves. These findings may be consistent with the actual area of the NVC. Hemodynamic features of the site of NVC can be added to the preoperative simulation for MVD surgery, which may be useful for the diagnosis and treatment planning of TN and HFS.

https://thejns.org/doi/abs/10.3171/2018.1.JNS172544

KEYWORDS computational fluid dynamics; hemifacial spasm; neurovascular contact; trigeminal neuralgia; wall shear stress; pain

$\mathrm{S}$ YMPTOMS of neurovascular compression syndromes such as trigeminal neuralgia (TN) and hemifacial spasm (HFS) are caused by neurovascular contact (NVC) between blood vessels and nerves. ${ }^{2,4}$ Microvascular decompression (MVD) surgery is usually carried out to release the contact of vessels from nerves. ${ }^{5,8-10}$ The patho- physiological mechanism underlying the causal relationship between blood vessels and nerves in NVCs is not fully elucidated. In the present study, we used computational fluid dynamics (CFD) to assess the hemodynamic characteristics of the offending vessels at NVCs. We investigated specific parameters at NVCs including the magnitude and

ABBREVIATIONS AICA = anterior inferior cerebellar artery; CFD = computational fluid dynamics; FR-avg = time-averaged flow rate; HFS = hemifacial spasm; MVD = microvascular decompression; NVC = neurovascular contact; NVC-d = distal NVC area; NVC-j = just-beginning NVC area; NVC-p = proximal NVC area; NVC-s = contact site NVC area; PICA = posterior inferior cerebellar artery; SCA = superior cerebellar artery; TN = trigeminal neuralgia; WSS = wall shear stress; WSSm = magnitude of WSS; WSSm-avg = time-averaged WSSm; WSSm-d = WSSm at distal area of NVC; WSSm-dia = end-diastolic WSSm; WSSm-j = WSSm at just-beginning area of NVC; WSSm- $=$ WSSm at proximal area of NVC; WSSm-s = magnitude of WSS at contact site; WSSm-sys = peak-systolic WSSm; WSSmV = temporal variation of magnitude of WSS; WSSv = direction of WSS; WSSVV = temporal variation of direction of WSS.

SUBMITTED October 8, 2017. ACCEPTED January 16, 2018.

INCLUDE WHEN CITING Published online July 6, 2018; DOI: 10.3171/2018.1.JNS172544. 
direction of wall shear stress (WSSm and WSSv, respectively) and their temporal variations in a single cardiac cycle (WSSmV and WSSvV). These parameters were compared with the simulation images and operative findings. To our knowledge, the relevance of hemodynamic features of the offending vessels to the morphological findings of the affected cranial nerves at NVCs has not been reported.

\section{Methods}

This retrospective study was approved by the ethics committee of Satoh Neurosurgical Hospital. Written informed consent was obtained from all patients for the use of their clinical data.

\section{Patient Population}

Between January 2015 and September 2016, 20 patients with neurovascular compressive diseases underwent MVD at our hospital (Table 1). Of the 20 patients, 10 had TN (5 men and 5 women) and 10 had HFS ( 2 men and 8 women). The median age of the TN patients was 66.5 years (range 37-85 years). The neuralgia was on the right side in 7 patients and the left side in 3 patients. The offending vessel was the trunk of the superior cerebellar artery (SCA) in 3 patients and a caudal branch of the SCA in 7 patients. The median age of the patients in the HFS group was 60.0 years (39-74 years), and the affected side was the right in 2 patients and the left in 8 patients. The offending vessel was the posterior inferior cerebellar artery (PICA) in 9 patients and the anterior inferior cerebellar artery (AICA) in the remaining patient.

\section{Imaging Procedure of Preoperative 3D CT Angiography}

A multidetector CT system (Activion-16, Canon Medical Systems) was used in Real-Prep scan mode with $100 \mathrm{ml}$ of contrast agent (Iomeron, iodine concentration $350 \mathrm{mg} / \mathrm{ml}$, Eizai Pharmaceutical) injected at $3 \mathrm{ml} / \mathrm{sec}$ into the antecubital vein. Imaging parameters were 16 $\mathrm{cm}$ field of view, a $512 \times 512$ matrix, 0.5 -mm thickness, scanning time 8 seconds, and a total of 201 images. The original volume data were reconstructed into a matrix of $1024 \times 1024$ pixels and a thickness of $0.3 \mathrm{~mm}$ using a workstation (Ziostation-2, Ziosoft-AMIN). The vessel configurations were reconstructed by a volume-rendering algorithm, and the arterial lumen was selected from the opacity chart of the volume data with a threshold of $>90$ Hounsfield units (HU).

\section{CFD Analysis}

Patient-specific geometries were generated as a stereolithography format using the preoperative 3D CT angiography, including the proximal end of the intracranial vertebral arteries, the basilar artery, the PICAs, the AICAs, the SCAs, and the proximal segment of the posterior cerebral arteries (PCAs).

These surface meshes were filled with unstructured cells mainly composed of hexahedrons. The size of the meshes was adjusted to vary in space. In far-wall regions, the length of the meshes was approximately $0.25 \mathrm{~mm}$, and in near-wall regions the meshes were aligned to fit the
TABLE 1. Characteristics of the TN and HFS groups

\begin{tabular}{ccc}
\hline Characteristic & TN $(n=10)$ & HFS $(n=10)$ \\
\hline Age, yrs & & 60.0 \\
\hline Median & 66.5 & $54.25-64.5$ \\
\hline IQR & $61.25-70.25$ & $39-74$ \\
\hline Range (min-max) & $37-85$ & \\
\hline Sex & & 2 \\
\hline Male & 5 & 8 \\
\hline Female & 5 & 2 \\
\hline Side & & 8 \\
\hline Right & 7 & \\
\hline Left & 3 & \\
\hline Offending vessels & & \\
\hline SCA trunk & 3 & 1 \\
\hline SCA caudal & 7 & \\
\hline PICA & & \\
\hline AICA & & \\
\hline
\end{tabular}

Max = maximum; $\min =$ minimum .

Values are numbers of cases unless otherwise indicated.

boundary with 3 layers. In the area nearest the wall, the size of the mesh was $0.125 \mathrm{~mm}$ in width and $0.05 \mathrm{~mm}$ in height. Flow was computed in a 3D unsteady fashion using a commercial package (Hemoscope v1.4, EBM Corp., Tokyo). The blood was assumed to be incompressible and Newtonian fluid with a density of $1050 \mathrm{~kg} / \mathrm{m}^{3}$ and a viscosity of $0.004 \mathrm{~Pa}$-sec. The Euler and second-order upwind schemes were utilized for discretizing unsteady and convective acceleration terms. The time step was determined to keep the Courant number less than 1 . The boundary conditions were determined in accordance with a constant WSS theory $(1.5 \mathrm{~Pa})$. A pulsatile flow-rate waveform was added based on the work of Ford et al. ${ }^{3}$ A pulsatile pressure waveform was set as a physiological one with an average of $100 \mathrm{~mm} \mathrm{Hg}$. The inlet of the vessel was set as a pressure boundary and the outlet as a velocity boundary.

The vascular geometry was segmented including a proximal NVC area (NVC-p), the just-beginning NVC area (NVC-j), the contact site NVC area (NVC-s), and the distal NVC area (NVC-d). We analyzed the time-averaged flow rate (FR-avg) and WSSm of the peak-systolic (WSSm-sys), end-diastolic (WSSm-dia), and time-averaged (WSSm-avg) phases at the above segmented areas, respectively. The WSSm was detailed in a 3D time series. Likewise, the temporal variations of WSSm (WSSmV) and WSSv (WSSvV) were depicted in 3D.

\section{Comparison of 3D CFD Images With Morphological Findings}

We reconstructed the 3D multifusion images by compositing a coregistered 3D CT angiogram and a 3D MR cisternogam, as previously described. ${ }^{8-10}$ Briefly, an MR cisternogram with a heavily T2-weighted 3D fast spinecho sequence was reconstructed with a perspective volume-rendering algorithm. Offending vessels delineated by the $3 \mathrm{D} \mathrm{CT}$ angiogram were superimposed on the $3 \mathrm{D}$ MR cisternogram. The anatomical elements consisting of 
the NVC, including the offending vessels, affected nerves, and surrounding brain parenchyma, were depicted as a single 3D image. Postoperatively, 3D multifusion images were reconstructed to simulate the operative view.

The CFD features of the offending vessels in the vicinity of the NVC were analyzed and displayed in a surgical view and comparatively evaluated with the anatomical simulation of 3D multifusion images and operative photos. Comparison of images with respect to hemodynamic features, anatomical simulation, and operative findings was done by 3 independent neurosurgeons (T. Satoh, K.O., and M.K.). We were thus able to directly compare the WSS parameters at the various areas of each NVC to the morphological findings.

\section{Analysis of CFD Parameters}

As specific hemodynamic parameters at NVCs, we investigated the magnitude and direction of wall shear stress (WSSm and WSSv) and their temporal variations in a single cardiac cycle (WSSmV, WSSvV). We numerically assessed the WSSm in the individual cases by calculating the relative ratios of the WSSm at the NVC-j, NVC-s, and NVC-d areas to the NVC-p (i.e., the WSSm-j/WSSm-p ratio, the WSSm-s/WSSm-p ratio, and the WSSm-d/WSSmp ratio, respectively) (Fig. 1). The dynamic changes in the WSSm and WSSv were evaluated by using the animation display during a cardiac cycle in accord with the cardiac waveform. We used static images of the WSSvV at the NVC to assess the mobility of the vector in a single cardiac cycle, and we compared them with the morphological findings.

\section{Statistical Analysis}

The results of hemodynamic analysis are expressed as median values and interquartile ranges (IQRs). A MannWhitney U-test was used for assessing the statistical significance of differences between the WSSm ratios, independently. A $\mathrm{p}$ value $<0.05$ was considered to indicate statistical significance.

\section{Results}

\section{Numerical Value and Relative Ratio of WSSm}

The results of hemodynamic analysis are summarized in Table 2. The average flow rate (FR-avg) of the offending vessels varied among the individual patients. The WSSm values of the offending vessels at the NVCs were relatively high during the peak-systolic, end-diastolic, and cycle-average phases. The relative ratios of the WSSm in the individual cases revealed that the WSSm-j/WSSm-p ratio and the WSSm-s/WSSm-p ratio were both significantly higher than the WSSm-d/WSSm-p ratio $(\mathrm{p}<0.05)$.

\section{Animation and Static Images of WSSm and WSSv}

Temporal changes of WSSm and WSSv in a single cardiac cycle are shown in the animation in Video 1.

VIDEO 1. Animation and summary of the WSSm and WSSv at the NVC for a representative case of TN (Fig. 2). Copyright Toru Satoh.

Published with permission. Click here to view.

The animation of WSSm showed dynamic change of mag-

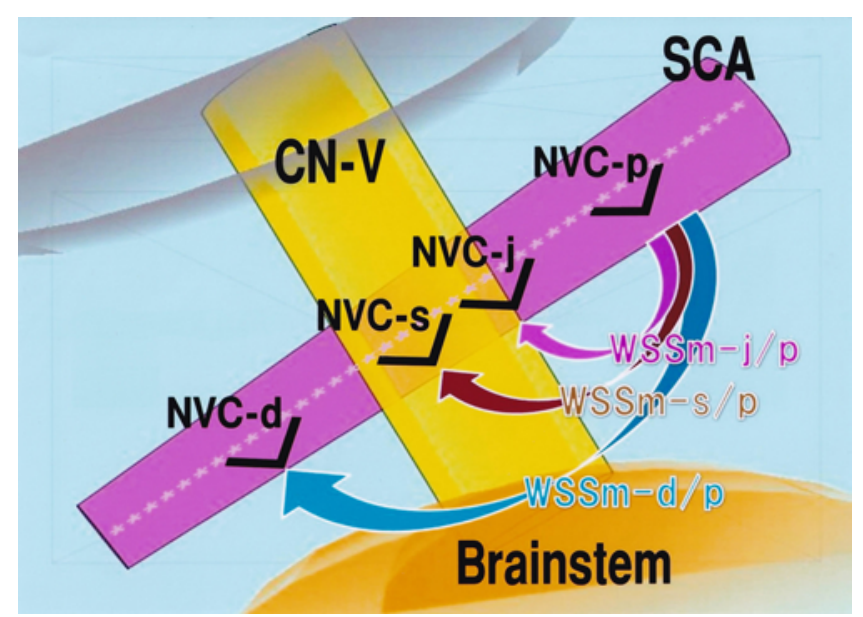

FIG. 1. Schema of NVC in TN. Segmentation of vessel: proximal area of NVC (NVC-p), just-beginning area of NVC (NVC-j), contact site of NVC (NVC-s), and distal area of NVC (NVC-d). Relative ratios of the WSSm compared to the WSSm at NVC-p: at NVC-j (WSSm-j/p ratio), NVC-S (WSSm-s/p ratio), and NVC-d (WSSm- $d / p$ ratio). $C N-V=$ trigeminal nerve.

nitude in accordance with a cycle waveform. The animation of the WSSv showed movement of the vector arrows during a single cardiac cycle. On the static images of $\mathrm{WSSvV}$, an area of small and stable movement of the vector was depicted on the vessel walls on the side in contact with the nerve. In contrast, large or unstable movements of the vector were observed on the vessel sides that were not in contact with the nerve. Representative cases of WSS in the CFD analysis for TN (Fig. 2) and HFS (Fig. 3) were shown.

\section{Discussion}

In patients with TN and HFS, the offending vessels usually compress the nerves severely. ${ }^{2,4,5,8-10}$ Nerves are compressed by vessels and may adhere to vessel walls, resulting in morphological changes such as deformation and dimple. At the NVC, the movable range of both the vessels and the nerves may be limited. Accordingly, hemodynamic features of the NVC may be related to the compression and immobility of the offending vessels and the affected nerves.

The WSS indicates the frictional force exerted by the flowing blood tangentially on the vessel wall during the cardiac cycle. The WSS is a vector quantity whose characteristics can be expressed by its magnitude (WSSm) and direction (WSSv). Temporal variation of the WSSv (WSS$\mathrm{vV}$ ) quantifies an accumulated amount of angular change of a single WSS vector over an entire cardiac cycle. This characteristic value can denote directional unsteadiness of the WSS.

\section{Hemodynamic Features of the NVC}

Two specific features of hemodynamic parameters were demonstrated at the NVCs of the offending vessels in patients with TN and HFS in this study. One feature was associated with the relative ratio of the WSSm, which showed that the magnitude of the WSS increased com- 
TABLE 2. Hemodynamic features of the TN and HFS cases

\begin{tabular}{|c|c|c|c|c|}
\hline Group \& Feature & NVC-p & $N V C-j$ & NVC-s & NVC-d \\
\hline \multicolumn{5}{|l|}{$\operatorname{TN}(n=10)$} \\
\hline \multicolumn{5}{|l|}{ FR-avg, $\mathrm{ml} / \mathrm{min}$} \\
\hline Median & 17.17 & 17.11 & 17.10 & 17.11 \\
\hline IQR & $9.63-28.10$ & $9.60-28.11$ & $9.65-27.94$ & $9.62-28.11$ \\
\hline \multicolumn{5}{|l|}{ WSSm-sys, $\mathrm{Pa}$} \\
\hline Median & 2.55 & 6.05 & 2.90 & 2.15 \\
\hline IQR & $1.58-4.04$ & $3.72-8.27$ & $2.12-7.82$ & $1.29-3.88$ \\
\hline \multicolumn{5}{|l|}{ WSSm-dia, Pa } \\
\hline Median & 0.96 & 2.34 & 1.19 & 0.79 \\
\hline IQR & $0.63-1.48$ & $1.48-3.08$ & $0.84-2.83$ & $0.54-1.41$ \\
\hline \multicolumn{5}{|l|}{ WSSm-avg, $\mathrm{Pa}$} \\
\hline Median & 1.48 & 3.64 & 1.80 & 1.27 \\
\hline IQR & $0.96-2.36$ & $2.26-4.90$ & $1.28-4.58$ & $0.79-2.28$ \\
\hline WSSm-sys, ratio & Ref & & & \\
\hline Median & & $1.93^{* *}$ & $1.29 *$ & 0.77 \\
\hline IQR & & $1.45-2.22$ & $1.05-1.81$ & $0.69-0.98$ \\
\hline WSSm-dia, ratio & Ref & & & \\
\hline Median & & $1.89 *$ & $1.21^{* *}$ & 0.76 \\
\hline IQR & & $1.34-2.22$ & $1.05-1.78$ & $0.68-0.93$ \\
\hline WSSm-avg, ratio & Ref & & & \\
\hline Median & & $1.69 *$ & $1.26^{\star *}$ & 0.84 \\
\hline IQR & & $1.38-2.06$ & $1.02-1.83$ & $0.73-1.26$ \\
\hline \multicolumn{5}{|l|}{ HFS $(n=10)$} \\
\hline \multicolumn{5}{|l|}{ FR-avg, $\mathrm{ml} / \mathrm{min}$} \\
\hline Median & 29.64 & 24.12 & 23.96 & 24.08 \\
\hline IQR & $16.26-39.81$ & $17.03-38.40$ & $17.37-38.47$ & $17.29-38.83$ \\
\hline \multicolumn{5}{|l|}{ WSSm-sys, $\mathrm{Pa}$} \\
\hline Median & 2.58 & 5.23 & 3.85 & 2.71 \\
\hline IQR & $2.27-5.83$ & $3.24-6.79$ & $3.41-5.88$ & $2.49-4.59$ \\
\hline \multicolumn{5}{|l|}{ WSSm-dia, $\mathrm{Pa}$} \\
\hline Median & 0.96 & 1.74 & 1.57 & 1.12 \\
\hline IQR & $0.81-1.86$ & $1.34-2.40$ & $1.31-2.17$ & $0.93-1.72$ \\
\hline \multicolumn{5}{|l|}{ WSSm-avg, $\mathrm{Pa}$} \\
\hline Median & 1.54 & 2.98 & 2.87 & 1.75 \\
\hline IQR & $1.35-3.29$ & $2.22-3.93$ & $2.10-3.53$ & $1.49-2.73$ \\
\hline WSSm-sys, ratio & Ref & & & \\
\hline Median & & $1.80^{*}$ & $1.43^{*}$ & 1.00 \\
\hline IQR & & $1.26-2.18$ & $1.27-1.52$ & $0.77-1.13$ \\
\hline WSSm-dia, ratio & Ref & & & \\
\hline Median & & $1.73^{* *}$ & $1.49^{*}$ & 1.11 \\
\hline IQR & & $1.33-2.06$ & $1.30-1.61$ & $0.88-1.23$ \\
\hline WSSm-avg, ratio & Ref & & & \\
\hline Median & & $1.28^{* *}$ & $1.45^{\star}$ & 1.05 \\
\hline IQR & & $1.22-1.78$ & $1.29-1.53$ & $0.91-1.23$ \\
\hline
\end{tabular}

Avg = average; dia = diastolic; NVC = neurovascular contact; NVC- $d$ = distal area of NVC; NVC - = just-beginning area of NVC; NVC-p = proximal area of NVC; NVC-s = NVC contact site; sys = systolic; WSSm = magnitude of wall shear stress.

${ }^{*} p<0.05,{ }^{* *} p<0.01$ by Mann-Whitney U-test of the WSSm ratio for NVC-j/NVC- $p$, NVC-s/NVC- $p$, and NVC-d/NVC- $p$.

paratively along the NVC, including the proximal, justbeginning, contact site, and distal areas of the NVC. The other feature was associated with the static image of the
WSSvV, which showed that the temporal variation of the WSS was decreased at the site of NVC.

The WSSm at the NVC area showed relatively high 


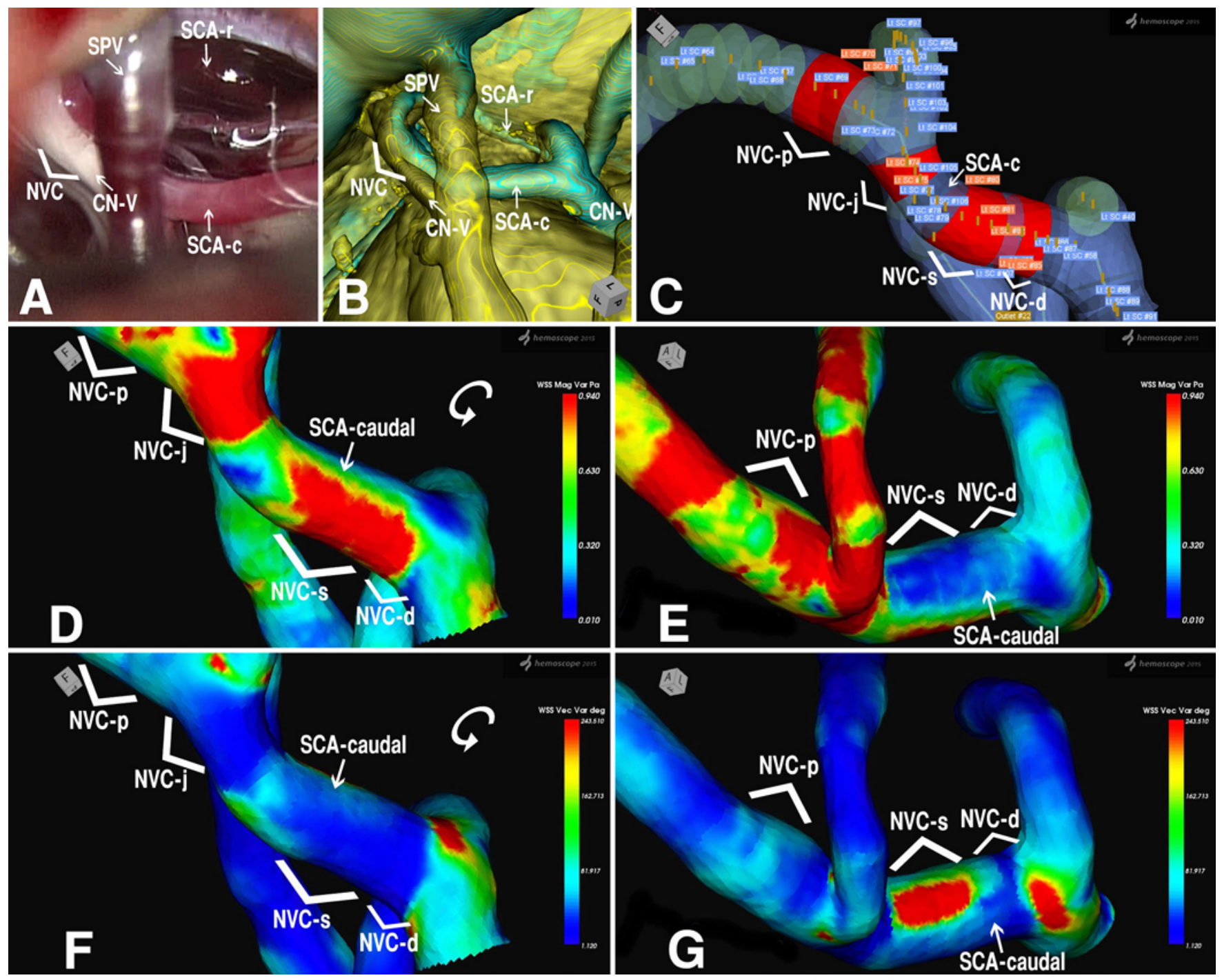

FIG. 2. Visualization of wall shear stress (WSS) in the CFD analysis of a representative case of TN, with an intraoperative photograph (A), simulated multifusion image (B), simulated and segmented vascular geometry of CFD of the ipsilateral projection in contact with the nerve $(\mathbf{C})$, the ipsilateral projection of temporal variation in the magnitude of the WSS in contact with the nerve (D), the contralateral projection of temporal variation in the magnitude of WSS $(E)$, the ipsilateral projection of temporal variation in the vector of the WSS $(\mathbf{F})$, and the contralateral projection of temporal variation in the vector of WSS $(\mathbf{G})$. NVC = area of neurovascular contact; NVC-d = distal area of NVC; NVC-j = just-beginning area of NVC; NVC-p = proximal area of NVC; NVC-s = contact site of NVC; SCA-C = caudal segment of SCA; SCA-r = rostral segment of SCA; SPV = superior petrosal vein.

values during the peak-systolic, end-diastolic, and cycleaverage phases. The animation display depicted the dynamic changes in the numerical value of the WSSm along the NVC in a cardiac cycle. Moreover, the WSSm values at the NVC- $j$ and NVC-s were higher than those at the NVC$p$ and NVC-d. The ratios of the WSSm at the NVC-j and NVC-s to the NVC-p (i.e., the WSSm-j/WSSm-p ratio and WSSm-s/WSSm-p ratio) were significantly higher than the WSSm-d/WSSm-p ratio $(p<0.05)$. These observations indicated that the magnitude of the WSS of the offending vessels increased at the area of the NVC. Though patients with TN and HFS experience different clinical symptoms, the above hemodynamic features can be commonly observed in both conditions. A relatively high shear stress at the NVC may cause arteriosclerotic remodeling of the vessels as a mechanotransduction response of vascular endothelial cells. ${ }^{1,6}$ Also, a contact of the offending vessels with affected nerves may deform the luminal geometry. We speculate that these two factors may be, at least in part, responsible for increased WSS.

In the animated display of WSSv, the dynamic change in the directions of WSS exhibited various types of flow motions including parallel, rotational, convergent patterns. Relatively small or stable movements were observed at the side of the offending vessel that was in contact with the nerve. Areas of less movement of WSSv at the contacting side were well differentiated from other areas of the NVC. In contrast, frequent or unstable vector movements were depicted on the vessel wall at the sides lateral and contralateral to the nerve. Because the WSSvV showed 


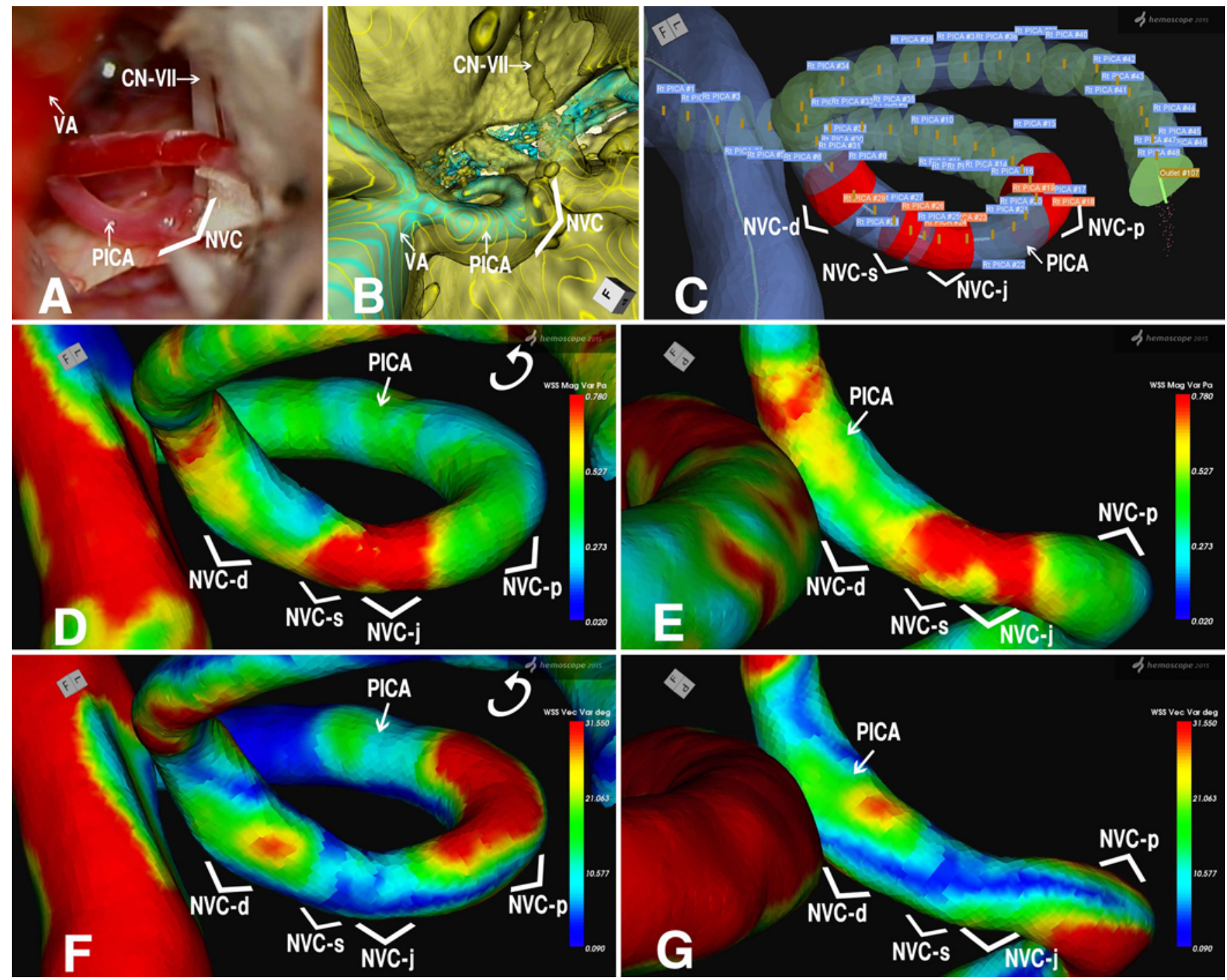

FIG. 3. Visualization of the WSS in the CFD analysis of a representative case of HFS, with an intraoperative photograph (A), simulated multifusion image (B), simulated and segmented vascular geometry of CFD of the ipsilateral projection in contact with the nerve (C), the ipsilateral projection of temporal variation in the magnitude of the WSS in contact with the nerve (D), the contralateral projection of temporal variation in the magnitude of WSS (E), the ipsilateral projection of temporal variation in the vector of the WSS $(\mathbf{F})$, and the contralateral projection of temporal variation in the vector of WSS (G). CN-VII = facial nerve; VA = vertebral artery.

an accumulated angular change of WSSv, distributions of WSSvV may suggest a range of mobility of the vessels. Consequently, stable WSSvV depicted at the area in contact with the affected nerve may indicate a limitation of movement of the offending vessel. Simultaneously, the mobility of the affected nerve may be reduced at the area of the NVC.

In general, curved vessel geometry leads to eccentric flow. The eccentric flow results in a local flow acceleration that increases the magnitude of WSS. As for the temporal variation of WSS, then, the outer and inner walls of the vessel, respectively, undergo stable and unstable movements due to an acceleration and deceleration. These flow features arise in stenotic vessels as well, where the proximal to middle part of the site of NVC experiences higher magnitude and stable variation of WSS, whereas the middle to distal region experiences the opposite. Our findings of hemodynamic features at the NVC can be explained accordingly. Thus, the spatial changes of WSS that we observed reflected an NVC-induced vascular deformation. Such a small deformation may often be difficult to identify geometrically, but the hemodynamic features demonstrated by CFD may help to assess the location and degree of NVC.

\section{Limitation of a CFD Analysis of NVC}

Several limitations may exist in the evaluation and analysis of hemodynamic characteristics in cases of TN and HFS. First, because the offending vessels, such as the SCA and its tributaries, the AICA, and the PICA, are small in size, it is difficult to create patient-specific geometries of vessels in detail in some cases. A second limitation is related to the reconstruction of the image data with the use of the volume-rendering technique. In the process of 
selecting the offending vessels and excluding others, there may be difficulties in separation of the arteries, veins, and surrounding bony structures. The third limitation concerns the matching of the location of the NVC between the 3D CFD images and the anatomical simulated images. The 3D simulated images are reconstructed by a perspective volume-rendering algorithm, whereas the 3D CFD images are created by a parallel volume-rendering algorithm. A certain incompatibility exists between these algorithms. A fourth limitation concerns the assessment of the static WSSvV image. Though the exact area of a small variation in an NVC is well recognized visually, the real extent and degree of compression at the NVC must be compared and confirmed by the actual operative findings.

\section{Benefits and Perspectives of CFD Analysis of NVCs}

In the neurosurgical MVD operation for TN and HFS, offending vessels compressing the nerves should be isolated and moved to eliminate physical contact with the nerve. Hemodynamic features of the offending vessels at NVCs may be useful for treatment planning of MVD. Although anatomical condition may differ in the TN and HFS, the CFD analysis may reveal similar results and tendencies at the NVCs. Patterns of the changes in the relative WSSm ratios along the vessels may correlate with the NVC region. In addition, the small variation revealed by the WSS$\mathrm{vV}$ may be related to the areas of restricted movement of the offending vessels at the NVC, as well as the affected nerves contacted and adhered by the vessels.

Prospectively, in combination with the preoperative anatomical simulation of 3D multifusion images, hemodynamic features of the WSS may play a role in the evaluation of the specific areas, especially the just-beginning areas and contact sites of NVCs for MVD surgery. More work is required to validate the imaging techniques described herein and to clarify the significance of hemodynamic features detected by CFD regarding the pathophysiology of NVCs for the execution of MVD surgery in patients with neurovascular compression syndromes.

\section{Conclusions}

Patterns of the changes in the relatively high WSSm ratio along the vessels may correlate with the NVC regions. Small variation in the WSSvV may be related to the areas of movement limitation at the NVC of the offending vessels. Hemodynamic features of the WSS may play a role in the preoperative evaluation of the specific areas of NVCs in patients with TN and HFS.

\section{Acknowledgments}

We wish to express our appreciation of Ms. Megumi Sasaki and Mr. Masato Miyauchigo, radiological technicians, and Ms. Kana Murakami, laboratory medical technologist, Ryofukai Satoh Neurosurgical Hospital, for conducting CT and MR examinations.

\section{References}

1. Ando J, Yamamoto K: Vascular mechanobiology: endothelial cell responses to fluid shear stress. Circ J 73:1983-1992, 2009

2. DeSouza DD, Hodaie M, Davis KD: Structural magnetic resonance imaging can identify trigeminal system abnormalities in classical trigeminal neuralgia. Front Neuroanat 10:95, 2016
3. Ford MD, Alperin N, Lee SH, Holdsworth DW, Steinman DA: Characterization of volumetric flow rate waveforms in the normal internal carotid and vertebral arteries. Physiol Meas 26:477-488, 2005

4. Haller S, Etienne L, Kövari E, Varoquaux AD, Urbach H, Becker M: Imaging of neurovascular compression syndromes: Trigeminal neuralgia, hemifacial spasm, vestibular paroxysmia, and glossopharyngeal neuralgia. AJNR Am J Neuroradiol 37:1384-1392, 2016

5. Leal PRL, Hermier M, Souza MA, Cristino-Filho G, Froment JC, Sindou M: Visualization of vascular compression of the trigeminal nerve with high-resolution 3T MRI: a prospective study comparing preoperative imaging analysis to surgical findings in 40 consecutive patients who underwent microvascular decompression for trigeminal neuralgia. Neurosurgery 69:15-26, 2011

6. Malek AM, Alper SL, Izumo S: Hemodynamic shear stress and its role in atherosclerosis. JAMA 282:2035-2042, 1999

7. Reymond P, Merenda F, Perren F, Rüfenacht D, Stergiopulos $\mathrm{N}$ : Validation of a one-dimensional model of the systemic arterial tree. Am J Physiol Heart Circ Physiol 297:H208H222, 2009

8. Satoh T, Omi M, Nabeshima M, Onoda K, Date I: Severity analysis of neurovascular contact in patients with trigeminal neuralgia: assessment with the inner view of the 3D MR cisternogram and angiogram fusion imaging. AJNR Am J Neuroradiol 30:603-607, 2009

9. Satoh T, Onoda K, Date I: Fusion imaging of three-dimensional magnetic resonance cisternograms and angiograms for the assessment of microvascular decompression in patients with hemifacial spasms. J Neurosurg 106:82-89, 2007

10. Satoh T, Onoda K, Date I: Preoperative simulation for microvascular decompression in patients with idiopathic trigeminal neuralgia: visualization with three-dimensional magnetic resonance cisternogram and angiogram fusion imaging. Neurosurgery 60:104-114, 2007

\section{Disclosures}

The authors report no conflict of interest concerning the materials or methods used in this study or the findings specified in this paper.

\section{Author Contributions}

Conception and design: Satoh, Onoda, Kameda, Date. Acquisition of data: Satoh, Onoda, Kameda, Sasaki, Ichikawa. Analysis and interpretation of data: all authors. Drafting the article: Satoh, Date. Critically revising the article: all authors. Reviewed submitted version of manuscript: all authors. Approved the final version of the manuscript on behalf of all authors: Satoh. Statistical analysis: Satoh. Administrative/technical/material support: Satoh. Study supervision: Date.

\section{Supplemental Information \\ Videos}

Video 1. https://vimeo.com/258989450.

\section{Previous Presentations}

Portions of this work were presented at the 13th International Conference on Cerebrovascular Surgery, October 29, 2017, Nagoya, Japan.

\section{Current Affiliations}

Dr. Onoda: Teikyo University Hospital, Tokyo, Japan.

\section{Correspondence}

Toru Satoh: Ryofukai Satoh Neurosurgical Hospital, Hiroshima, Japan.ucsfbtrc@urban.ne.jp. 